\title{
Une interprétation institutionnaliste des deux grandes crises de la mondialisation (critiquer la société de marché avec Karl Polanyi)
}

\author{
Jérôme Maucourant \\ Université Jean Monnet, Saint-Étienne, France
}

\section{INTRODUCTION}

La crise actuelle, qui a commencé durant

l'été 2007, a de multiples dimensions. Crise morale, d'abord, parce que l'esprit de démesure, qui gouverne le monde de l'argent, rencontre une désapprobation sociale croissante. Crise politique, ensuite, car la démocratie semble impuissante à régler l'économie au bénéfice du bien commun. Crise économique, enfin, car les conceptions théoriques et pratiques en ce domaine sont frappées d'une évidente obsolescence.

La crise actuelle, qui a commencé durant l'été 2007, a de multiples dimensions. Crise morale d'abord, parce que l'esprit de démesure, qui gouverne le monde de l'argent, rencontre une désapprobation sociale croissante. Crise politique ensuite, car la démocratie semble impuissante à régler l'économie au bénéfice du bien commun. Crise économique, enfin, car les conceptions théoriques et pratiques en ce domaine sont frappées d'une évidente obsolescence. Néanmoins, la mise en perspective historique et l'art raisonné de la comparaison sont des éléments utiles pour la compréhension des phénomènes les plus contemporains, et cette compréhension est une condition nécessaire pour toute action à l'efficacité durable. L'approche comparative proposée ici recoupe une démarche institutionnelle: tout n'est pas possible en effet et l'héritage en termes d'institutions pèse sur les choix politiques. À cet égard, rappelons que la vie sociale repose sur des ensembles idéologiques et normatifs - les institutions - qui expriment des compromis sociaux et des préférences collectives. Nous ne sommes donc pas, toutefois, prisonniers des tendances lourdes héritées du mouvement historique, car la connaissance même des institutions qui sont autant de déterminations socio-historiques est à la source des succès de l'invention politique.

Il se trouve que nos difficultés contemporaines se prêtent assez bien à l'entreprise de comparaison : les turbulences de l'été 2007 ont manifesté la seconde crise de la mondialisation, la première s'étant produite en 1929. Il va de soi que les conditions technique, écologique et culturelle ont beaucoup changé, mais il demeure un trait commun à ces deux époques, une idée étrange du point de vue de la longue durée de l'aventure humaine et si propre à la modernité occidentale, c'est-à-dire la soumission volontaire de la société à un système de marchés autorégulateurs. Le sens même de la politique libérale au XIX ${ }^{\mathrm{e}}$ siècle fut d'immerger la société dans l'économie, alors que les systèmes socio-économiques connus jusqu'alors encastraient l'économie dans la société. Le « néolibéralisme », sous la houlette américaine, n'a fait que réactualiser, au moment du déclin du soviétisme à la fin des années 1970, ce projet qui fut d'abord celui de l'Empire britannique il y a un siècle et demi. Nous montrerons que le capitalisme a besoin de politique pour naître et se perpétuer, la monnaie étant une institution essentielle à ce processus

Dans ce dessein, nous exposerons, en premier lieu, la nature de la société de marché, qui transforme fictivement en marchandises ce qui n'a pas été produit pour être vendu, comme la terre, le travail et la monnaie. Nous insisterons, en second lieu, sur le rôle que joue la monnaie comme institution dans la dynamique et l'impasse de la société de marché. Finalement, le marché autorégulateur semble bien être une utopie ou, plus précisément, un non-lieu, ce qui rend souhaitable son dépassement du point de vue d'une vie bonne. 


\section{LA « SOCIÉTÉ DE MARCHÉ » COMME POLITIQUE}

Dans le cadre de la société de marché telle qu'elle se constitue, il y a deux siècles, en Occident, la « dépendance de l'homme visà-vis des biens matériels », via « la peur de la faim et l'espérance du gain », devient le moteur essentiel de la production.

Dans la plupart des sociétés humaines, les motivations, qui conduisent les hommes à produire de façon à assurer leurs conditions matérielles d'existence, sont la conséquence d'un certain nombre d'obligations sociales liées à la parenté, l'honneur, au fonctionnement de la hiérarchie sociale, voire, en certains cas, à la rivalité politique, l'esthétique ou la religion. Le système économique est donc généralement enchâssé (embedded) dans les relations sociales ${ }^{2}$. Il en va bien différemment dans nos sociétés.

\section{Les «marchandises fictives » et le cas de la monnaie}

Dans le cadre de la société de marché telle qu'elle se constitue, il y a deux siècles, en Occident, la « dépendance de l'homme vis-à-vis des biens matériels », via « la peur de la faim et l'espérance du gain $»^{3}$, devient le moteur essentiel de la production. Ce fait nouveau, déjà souligné par Marx et Weber, est lié à la marchandisation d'un certain nombre de relations sociales.

À la fin de l'ère féodale, en effet, il se constitue progressivement un marché de la terre. Ceci implique que la nature, qui n'est pas produite pour être vendue comme l'est la marchandise, soit traitée sous le nom de «terre » comme une marchandise : c'est là une pure fiction. Quelques siècles plus tard, à l'aube de la révolution industrielle, l'homme, sous le nom de « travail», est traité pareillement, c'est-à-dire in fine comme «marchandise fictive». Le fait des marchés de produits est une chose ancienne mais, comme l'on doit le remarquer, le fait que les «facteurs de production »- l'homme et la nature - rentrent dans le monde de la marchandise est le signe d'une révolution : la société est dès lors enchâssée dans le système économique. C'est pourquoi la «société de marché », que
l'Empire britannique mondialise au $\mathrm{XIX}^{\mathrm{e}}$ siècle, est singulière en ce que qu'aucune autre société n'a auparavant institué la peur de la famine et l'appât du gain comme motivations déterminantes de la production.

Bien sûr, la monnaie nous semble liée organiquement au monde marchand et, pourtant, elle est également une marchandise aussi fictive que la terre ou le travail, car la monnaie n'est pas produite pour être vendue. Nous savons, grâce aux découvertes anthropologiques du $\mathrm{XX}^{\mathrm{e}}$ siècle, que des sociétés ont pu disposer de systèmes monétaires hautement raffinés alors que les marchés n'existaient pas ou étaient insignifiants : la raison en est que la monnaie règle des obligations statutaires fort complexes dans ces sociétés, lesquelles ne sont pas de nature ou d'origine économique. Ainsi, les tributs, les amendes, les impôts, mais également tout ce qui est nécessaire pour accomplir les exigences des relations entre groupes, et enfin les exigences sacrificielles, sont à l'origine de la mobilisation de la monnaie comme moyen de paiement. La monnaie a donc une dimension symbolique, c'est-à-dire d'alliance.

Par ailleurs, quand l'origine économique des pratiques monétaires semble évidente, via l'endettement, c'est l'extrême précarité des conditions de vie qui pousse à des prêts de subsistance : l'esprit d'entreprise n'est pas la cause essentielle de l'endettement. La monnaie est donc d'abord, comme unité de compte, un moyen de codification sociale, qui donne une mesure institutionnelle des obligations entre des hommes entre eux ou entre ceux-ci et les dieux; ensuite, comme moyen de paiement, elle apaise ainsi les relations, comme l'indique l'étymologie. La société de marché s'est emparée de cette institution plurimillénaire, largement prémarchande, pour en faire un moyen d'échange.

La question se pose dès lors à savoir si notre modernité a vraiment expulsé toutes les dimensions politique et symbolique de l'institution de la monnaie. Ceci justifierait ainsi l'idée d'enlever au pouvoir politique toute forme d'influence en matière monétaire; ceci justifierait aussi 
d'imprimer des billets européens de banque centrale, d'où sont bannis toutes les références à des hommes, des événements ou des monuments renvoyant à la culture européenne. Autrement dit, même si nous savons, au moins depuis Engels et Marx, que la société de marché est l'empire des « eaux glacées du calcul égoïste », cela permet-il de fabriquer une société?

La réponse est clairement négative, dès le moment où régna une monnaie mondiale, l'étalon-or, dont l'appellation semblait suggérer que la monnaie n'était qu'une marchandise plus échangeable que les autres, à un point telle qu'elle était la liquidité même. Polanyi écrit, en effet, à ce sujet : " Or la séparation institutionnelle des sphères politique et économique n'a jamais été complète, et c'est précisément en matière de monnaie qu'elle a été nécessairement incomplète; l'État, dont la Monnaie semblait simplement certifier le poids des pièces, était en fait le garant de la valeur de la monnaie fiduciaire qu'il acceptait en règlement des impôts et autres paiements. Non, cette monnaie n'était pas un moyen d'échange, c'était un moyen de paiement; ce n'était pas une marchandise, c'était un pouvoir d'achat; (...) elle était simplement un symbole (nous soulignons) incorporant un droit quantifié à des choses qui pouvaient être achetées ${ }^{4}$.

Aujourd'hui, l'opération qui vise à transformer des dettes privées douteuses en dettes de l'État car la monnaie qui a cours légal est une dette de l'État - ne pose pas de problème insurmontable dans des sociétés $^{5}$, comme les États-Unis d'Amérique, qui se pensent comme communauté politique. Et pourtant, une telle monétisation devrait poser des problèmes sans fin si la monnaie n'était réellement qu'une image de la marchandise, comme le croient les tenants du néolibéralisme européen. D'ailleurs, le premier créditeur du Trésor américain, aujourd'hui, n'est plus la Chine mais le Système de réserve fédérale... une institution américaine!

\section{Le « double mouvement »}

Comme l'a illustré le cas de la monnaie de la Belle Époque, la séparation entre l'économie et la politique est donc une illusion. Il n'en reste pas moins que cette croyance utopique dans un fonctionnement autonome de la sphère économique produit des «effets de réalité », pour reprendre une expression de Pierre Bourdieu. Notons cependant que les comportements, croyances ou institutions, qui résultent de cette utopie, n'engendrent pas nécessairement une société viable. Traiter comme marchandise des entités, qui n'en sont pas, conduit nécessairement à des effets pervers, impliquant des formes d'autoprotection de la société. Une dialectique incertaine naît de ce mouvement de marchandisation, auquel répondent des contre-mouvements de protection-institutionnalisation nécessaires pour la perpétuation de la nature (la terre), l'homme (le travail) et la monnaie (la société) ${ }^{6}$ : le double-mouvement. Les politiques visant à la «libération» des «forces du marché » se heurtent à l'évidence suivante: "la prétendue marchandise qui a nom «force de travail» ne peut être bousculée, employée à tort et à travers, ou même laissée inutilisée, sans que soit également affecté l'individu humain qui se trouve être le porteur de cette marchandise particulière $»^{7}$.

Quant à la monnaie, il est frappant de constater qu'en dépit de rêveries idéologiques relatives aux monnaies concurrentielles, qui ont cours jusqu'à aujourd'hui, les crises à répétition ont entraîné, aux États-Unis mêmes en 1913 alors qu'ils furent longtemps réticents, l'institution d'un monopole d'émission et d'une véritable politique monétaire en 1919. Bien avant, les Britanniques avaient déjà rationalisé autant que possible le système de l'étalon-or; l'organisation consciente de la baisse des prix par l'élévation des taux d'intérêts qui permet de faire en sorte que la contrainte de convertibilitéor des monnaies nationales ne soit pas un désastre économique. À n'en pas douter, la liquidation des firmes les «moins efficaces » est le prix à payer pour cette politique ${ }^{8}$, mais la banque centrale nationale isole ainsi l'économie interne de chocs extérieurs dangereux. Parce que la politique monétaire est une forme de protection sociale, Polanyi écrit : «la protection sociale est l'accompagnement obligé d'un marché censé autorégulateur $»^{9}$. 
Quand le libéralisme en acte se heurte au réel, il se fait que, montent d'une manière imprévue, de toutes les strates sociales, des formes d'autoprotection de la société pouvant prendre la forme de compromis collectifs sanctionnés par l'État.

Quand le libéralisme en acte se heurte au réel, il se fait que, montent d'une manière imprévue, de toutes les strates sociales, des formes d'autoprotection de la société pouvant prendre la forme de compromis collectifs sanctionnés par l'État. Ceci rend le capitalisme tolérable et assurent paradoxalement, pour un temps, sa viabilité dans un espace donné. On comprend que le capitalisme soit condamné à une dilatation continue de son espace, de façon extensive ou intensive: la saturation de celui-ci l'annihilerait sous le poids des régulations qui sont sa paradoxale condition de possibilité. Mais, les contre-mouvements gênent peu à peu la capacité régulatrice du Grand Marché. Polanyi remarque, pour ce qui est de la fin du XIX ${ }^{\mathrm{e}}$ siècle : "les droits de douanes (...) les cartels et trusts, les embargos sur l'immigration (...) sont autant de freins pour le fonctionnement du système concurrentiel (...) et nuisent gravement au mécanisme autorégulateur du marché ».

Mais, ne nous méprenons pas sur la nature de ces entraves aux mécanismes marchands: la suppression de celles-là ne peut abolir les tensions inhérentes à la société de marché, dans la mesure où ce qui rend cohérent et viable à moyen terme le capitalisme sont ces entraves mêmes. Ce raisonnement présuppose un type donné des contraintes technique, démographique et écologique qui s'imposent au capitalisme : toute modification de ces données peut redonner de l'élan ou déprimer la dynamique capitaliste. D'ailleurs, que dire des capacités prétendument «régulatrices» du marché, si elles impliquent que le coût du travail doive être abaissé au-dessous d'un niveau vital ou d'un certain niveau culturel, incompatible avec la dignité humaine? Il devient dès lors évident que l'autorégulation de l'économie, domaine social pensé comme autonome par les libéraux, est une chimère. Pour ce qui est de la période postérieure à la Grande Guerre, elle se traduit par une montée de la politique démocratique : il devient impossible, après 1929, de dévaloriser le travail humain comme on le faisait aux beaux jours du capitalisme libéral dont l'inhumanité fut justement dénoncée par Marx.

À ce stade du raisonnement, il convient de rappeler qu'aucune société ne peut survivre si ses fonctions politiques et économiques ne s'accordent pas en quelque façon. C'est pourquoi, déçues par une démocratie impuissante à régler l'ordre économique tenu par les propriétaires, les masses soutiennent de plus en plus l'idée fasciste, qui ne cesse de progresser à la fin des années $1920^{10}$ et atteint son acmé en 1933. Les fascismes constituent pour Polanyi une modalité de la "Grande Transformation », ce changement majeur qui met à bas le vieux capitalisme à régulation concurrentielle. De la contradiction, qui vire à l'antagonisme entre économie et politique, le fascisme peut se définir comme l'absorption de la politique par l'économie.

Polanyi écrit ainsi : «On peut décrire la solution fasciste à l'impasse ${ }^{11}$ où s'était mis le capitalisme libéral comme une réforme de l'économie de marché au prix de l'extirpation de toutes les institutions démocratiques $»^{12}$. Deux types d'explications sont mobilisées : du point de vue politique, l'opposition des libéraux à toute forme de dirigisme accélère la montée de la revendication autoritaire: «La privation totale de liberté dans le fascisme est, à vrai dire, le résultat inéluctable de la philosophie libérale qui prétend que le pouvoir et la contrainte sont le mal ${ }^{13}$. Le libéralisme, comme politique et comme représentation du monde, contient donc en lui-même la dérive fascisante, ce qu'il répugne souvent à reconnaître et ce qui explique la vive hostilité de certains milieux libéraux à l'encontre du travail de Polanyi. Il est évident que seule la violence peut engendrer un type de société qui liquide les droits politiques. Dans ces conditions : «les êtres humains sont considérés comme producteurs et producteurs seulement (...) La représentation est accordée à la fonction économique; elle est technique et impersonnelle ${ }^{14}$. Les corporations fascistes absorbent 
une bonne part de 1'«État politique » propre au système libéral traditionnel.

Mais, comment produire de la communauté dans ce monde fasciste qui est aussi déshumanisant qu'hypermoderne? N'oublions pas ce qu'écrit à ce sujet le grand historien allemand, Norbert Frei, «qui montre que les mentalités collectives ont été façonnées durant la période nazie : l'importance accordée au rôle du producteur a impliqué un "souci de rendement et de l'efficacité », « utile » lors de la reconstruction de l'Allemagne. Ce simple rappel montre à quel point l'inhumanité peut être inscrite dans les développements industriels issus de la société de marché, sur quoi Polanyi ne cesse d'insister au grand dam de nombre de libéraux $»^{15}$. Dans ce monde réifié radicalement, le fascisme allemand produit de l'être ensemble par l'exaltation de la race, comme d'autres formes totalitaires peuvent le faire avec la religion. Ce type d'idéologie tente de nier l'Histoire en retrouvant une pureté d'avant l'Histoire; la politique, entendue comme espace commun où les hommes peuvent discuter et construire leur destinée, est niée au nom d'une vie mythique où la conscience même des individus n'a plus sa place.

Le capitalisme, qui émerge de la Grande Transformation des années 1930, est bien moins libéral que le précédent. Un certain nombre de facteurs expliquent ces faits : la fragilité des économies européennes, qui n'auraient pas supporté le choc d'une libéralisation rapide des flux internationaux, et l'ampleur de la menace soviétique, qui n'était pas sans effet quant à la position du travail dans le rapport de force existant avec le capital. Il est clair que l'affermissement des droits sociaux et la croissance continue du salaire ont été des moyens cruciaux de lutte contre l'influence soviétique. Celle-ci se caractérisait par l'aspect relativement autocentré de la croissance, qui permit l'émergence d'un contrôle étatique. En Corée du Sud ou au Japon, seules des interventions gouvernementales marquées (diverses politiques de promotion et de protections des capitalismes nationaux) ont pu faire éclore la prospérité là où elle était stratégiquement utile.

En Corée du Sud ou au Japon, seules des interventions gouvernementales marquées (diverses politiques de promotion et de protections des capitalismes nationaux) ont pu faire éclore la prospérité là où elle était stratégiquement utile.

\section{LES CRISES DES SOCIÉTÉS DE MARCHÉ : UN POINT DE VUE MONÉTAIRE}

\section{L'évolution vers la seconde société de marché est, pour une part, une involution permise par un certain nombre de facteurs durant les trois décennies 1980-2010 : la \\ transnationalisation accrue des marchés, la mobilisation d'un salariat déqualifié au niveau mondial, le démantèlement des protections qui avaient rendu possible l'émergence de capitalismes dynamiques au Sud, la transition vers le capitalisme à l'Est, l'arrivée à maturité de nouvelles technologies de l'information et de la communication.}

La réussite même de l'ère keynésienne a permis paradoxalement une modification de type qualitatif permettant de réactualiser ce que Polanyi dénommait «l'idéologie réactionnaire de Wall
Street ${ }^{16}$. L'évolution vers la seconde société de marché est, pour une part, une involution permise par un certain nombre de facteurs durant les trois décennies 1980-2010: la transnationalisation accrue des marchés, la mobilisation d'un salariat déqualifié au niveau mondial, le démantèlement des protections qui avaient rendu possible l'émergence de capitalismes dynamiques au Sud, la transition vers le capitalisme à l'Est, l'arrivée à maturité de nouvelles technologies de l'information et de la communication. Ainsi, cet «inter-siècle » américain, pour reprendre une notion utilisée par Jacques Sapir, actualise les virtualités que contenait l'hégémonie américaine qui, en 1945, prétendait reprendre l'héritage britannique. Mais, comme ce fut le cas pour l'Empire Britannique, la crise provient de l'impossibilité, pour la société de 
marché, de satisfaire le paiement d'un ensemble de dettes qui sont, pourtant, la condition sine qua non de son existence. La monnaie apparaît bien alors comme une institution sociopolitique réglant des conflits politiques structurants.

\section{L'impasse de la première société de marché}

\section{L'année 2008 a signé la démesure de l'esprit capitaliste qui, dans sa forme financière et mondialisée, n'a trouvé de limite que dans l'effondrement. Sans l'intervention massive des États, dont les néolibéraux ne cessaient de regretter l'excessive importance, les conséquences humaines et économiques auraient été bien pires qu'en 1929, comme beaucoup s'accordent à le reconnaître.}

L'année 2008 a signé la démesure de l'esprit capitaliste qui, dans sa forme financière et mondialisée, n'a trouvé de limite que dans l'effondrement. Sans l'intervention massive des États, dont les néolibéraux ne cessaient de regretter l'excessive importance, les conséquences humaines et économiques auraient été bien pires qu'en 1929, comme beaucoup s'accordent à le reconnaître. Maintenant, l'accroissement des dettes publiques exprime, pour une bonne part, ce qu'il faut payer comme prix des errances de la finance et de la cupidité des intérêts dominants. A contrario, si les dettes privées n'avaient pas été massivement monétisées et si les dépenses publiques n'avaient pas pris le relais de l'effondrement de la demande privée, nous vivrions une déflation prolongée des prix et une dépression profonde de l'économie «réelle». Les années 1930 illustrèrent ce cas de figure : la pléthore de dettes contractées alors forcèrent à des ventes de liquidation rendue nécessaire par la pénurie de monnaie. La peur de l' « inflationnisme » fut une source du fascisme.

Relire Polanyi permet d'illustrer de façon assez fascinante l'homologie structurale des deux crises de ce système mondial qu'est la société de marché : de façon à maintenir le socle social du capitalisme libéral après la Grande Guerre, les pays européens instituent des créances de facto en faveur de diverses classes sociales sur l'économie.
Or, le retour à la parité-or d'avant-guerre implique une élévation énorme de la rente financière. Seule la montée de la dette permet alors, à court terme, de satisfaire cette demande accrue sur les richesses produites. C'est donc le crédit international, devenu plus réceptif qu'autrefois aux exigences politiques, qui reporte dans le temps les déséquilibres européens.

Mais, si l'élasticité nouvelle du système financier ajourne la résolution des problèmes structurels, elle ne les résout nullement. La montée de la dette permet, en effet, à de nombreux pays européens de ne pas rembourser leurs dettes antérieures et l'Amérique peut se faire quelque illusion sur la valeur de ses créances. Des deux côtés de l'Atlantique, il existe des bénéfices mutuels à court terme. Par le contrôle de l'immigration européenne et les droits de douane, les États-Unis jouissent d'un niveau de vie «incongrûment élevé », comme si le bénéfice extraordinaire des exportations américaines dues à la guerre ne pouvait pas, par principe, être rétrocédé. Il vaudrait mieux que l'Amérique abandonne ses créances de guerre, quitte à faire baisser ainsi le niveau de vie avec des prélèvements fiscaux. Une autre méthode serait le maintien de ses créances en contrepartie d'une politique d'immigration plus généreuse, laquelle ferait également baisser le niveau de vie moyen. Or, «l'Amérique a non seulement maintenu ses créances, mais aussi, pour les sauvegarder, a accordé à l'Europe d'énormes crédits nouveaux $»^{17}$.

Dans ces conditions, la politique anglaise consistant à céder aux exigences des rentiers à propos du cours de la livre britannique est inadéquate. On s'attache à pratiquer une réévaluation de la livre qui implique une baisse des prix intérieurs. Or, cette politique anglaise de la «monnaie forte» ne peut réussir car la politique de baisse des salaires montre son échec en 1926. Dès lors, pour éviter une baisse de la Livre Sterling, il faut que le dollar soit moins attractif. Le soutien de la Livre exige une différence de taux d'intérêt entre Londres et New York ${ }^{18}$ : c'est l'objectif de la Cheap Money Policy ( «la politique d'argent bon marché ») américaine de mai 1927. En conséquence, le déséquilibre anglais est transmis aux États-Unis. 
Même si l'inflation de la dette n'est plus encouragée en février 1928 par la politique monétaire, le processus d'endettement et de folie boursière est allé si loin que la crise de liquidation est inéluctable. Dès que l'Amérique cesse ses crédits, c'est le processus de liquidation qui s'enclenche, ce qui provoque la crise du crédit en 1931 et la crise monétaire de 1933.

En bref, Polanyi estime que la perpétuation de la guerre et le maintien de l'ordre social durant la paix ont imposé de nouveaux rapports de forces. Or, ceux-ci sont créateurs de dettes dont l'accumulation n'est pas compatible avec un bon fonctionnement de la convertibilité en or des monnaies. La politique de la puissance rentière du système, les États-Unis, a, en définitive, de graves conséquences : la volonté de maintenir un certain type de rente financière et de contrôler étroitement l'immigration précipite la crise globale.

\section{La crise de la seconde société de marché}

De la même façon que les libéraux des années 1930 accusaient le laxisme monétaire des années 1920 d'avoir engendré la crise de 1929, les néolibéraux prétendent que les maux économiques de notre temps seraient le fruit d'un manquement à la pleine logique capitaliste : l'obsession du plein emploi aurait politisé le capitalisme et entravé ses capacités d'autoajustement. Tout se passe, ainsi, comme si nous avions trouvé la clef du meilleur des mondes, dans les années 1990... Les néolibéraux raisonnent, en réalité, comme si les politiques d'argent bon marché et la prolifération de la dette ne s'inscrivaient pas dans une nécessité qui s'impose à l'élite : conserver un taux de croissance suffisant qui est la condition de possibilité de cette mondialisation inégalitaire dont se nourrit sa domination. Une autre nécessité s'impose à l'élite des années 1920, mais c'est bien, dans les deux cas, une même volonté politique d'imposer un cadre de domination globale qui a des conséquences économiques.

C'est ce point essentiel que nous voulons démontrer, qui implique que, si les États-Unis avaient tranché en faveur d'une protection sociale digne de leur puissance et renoncé aux facilités de la finance et du «libre-échange», cette folie du crédit n'aurait pas été nécessaire. Une des raisons actuelles qui a contraint à l'inflation de la dette, via des inégalités croissantes, est le libre-échange: celui-ci, notamment aux États-Unis, est porteur de déflation salariale, ce qui va à l'encontre de l'opinio communis des vingt dernières années ${ }^{19}$. Les fameux excédents chinois, contrepartie comptable d'une partie du déficit commercial américain, ne font qu'exprimer un mode d'accumulation désindustrialisant et financiarisé. La croissance américaine d'avant crise doit donc beaucoup aux «progrès » des techniques de la finance, occultant à court terme les conséquences d'un endettement excessif, et à la mondialisation, qui a permis de compenser, par la baisse des prix relatifs de certains biens importés, la tendance à la baisse de la demande, elle-même résultat inéluctable d'une redistribution des gains de productivité à une très mince couche sociale.

Les discours orthodoxes, souvent aussi hypocrites que rétrospectifs, fustigeant les mauvaises pratiques financières, masquent que celles-ci sont une composante décisive de cette mondialisation dont ils se font les chantres. Sans disséminer ses dettes dans le monde, sans rendre liquides ses créances, le capitalisme bancaire américain n'aurait pas pu développer son activité de prêt avec l'énergie qu'on sait. Sans ce marché financier si attractif parce qu'inventif, les ÉtatsUnis n'auraient pas bénéficié de toute l'épargne du monde, et jamais la croissance mondiale n'eût été suffisante, dans ce système-monde polarisé autour de l'étalon-dollar.

Comment peut-on dénoncer sérieusement l'aveuglement supposé de gouverneurs de banques centrales, alors que ceux-ci ne faisaient que rendre possible la dynamique capitaliste : à un moment critique, celle-ci nécessitait de la monnaie bon marché et des garanties étatiques au crédit hypothécaire qui facilitaient l'endettement. Ces gouverneurs n'ont pas de mandat pour expérimenter une stagnation économique, voire une dépression, assurés qu'ils seraient du bon fonctionnement de la «main invisible » sur le «long terme», car, dans le temps de cette expérience, ce sont les fondements de la société de marché qui auraient été ébranlés, voire 
détruits. Ce sont donc bien les contraintes globales du capitalisme réellement existant et non les erreurs prétendues d'un président du Federal Reserve System, jugé trop à l'écoute de la démocratie, qui ont configuré les paramètres de la politique monétaire. Déplorer la montée de la dette privée, comme le font les néolibéraux, en faisant comme si elle ne s'inscrivait pas dans les nécessités du système économique des années 1990-2000, revient à vouloir poursuivre la chimère de la société de marché sans que ne soit jamais payé le coût de sa perpétuation.

De ce point de vue, le schéma d'analyse que propose Polanyi pour comprendre la Grande Crise de 1929 est utile pour saisir certaines dimensions de l'effondrement de 2008 : la société de marché ne peut fonctionner sans des dettes, qui expriment sa condition sociale de possibilité ${ }^{20}$, et il n'y a aucune «main invisible» permettant aux antagonismes sociaux de se dissoudre dans l'économie. Seuls des compromis institutionnalisés, qui construisent diverses formes d'actions collectives et les mécanismes de marché, peuvent stabiliser les conflits de classes et d'autres intérêts sociaux, dans la perspective d'une vie viable. En réalité, les marchés ne fonctionnent pas dans un

\section{CONCLUSION}

Dans le monde occidental, la contradiction est patente entre démocratie (politique) et économie (capitaliste); c'est que Polanyi

affirmait aux débuts des années 1930, et c'est aussi ce que soutient l'ancien secrétaire au Travail de Clinton, Robert Reich.

Dans le monde occidental, la contradiction est patente entre démocratie (politique) et économie (capitaliste); c'est ce que Polanyi affirmait aux débuts des années 1930, et c'est aussi ce que soutient l'ancien secrétaire au Travail de Clinton, Robert Reich ${ }^{21}$. Les événements qui affectent l'Europe, notamment en Grèce, proviennent de cette contradiction, laquelle est poussée, ici, bien plus loin qu'elle ne l'est aux États-Unis. Les partisans de l'eurolibéralisme se satisfont sans doute de cette singulière démocratie sans souveraineté à l'Européenne, qui protègerait les droits formels en écartant les vide social et culturel, sans des institutions qui sont des legs de l'histoire et expriment des rapports de force. La finance de marché contemporaine a ainsi rendu possible une croissance, que l'inégalité de nos temps exige structurellement, mais selon des prises croissantes de risque. À court terme, cellesci ont engendré des profits privés considérables qui impliquaient, à moyen terme, toutefois, une crise grave et une importante socialisation des coûts. En 1929 comme en 2008, la finance de marché n'a pas vécu de l'air du temps et l'a fait payer au prix fort à toute la société.

\section{La société de marché ne peut fonctionner sans des dettes, qui expriment sa condition sociale de possibilité, et il n'y a aucune « main invisible » permettant aux antagonismes sociaux de se dissoudre dans l'économie. Seuls des compromis \\ institutionnalisés, qui construisent diverses formes d'action collective et les mécanismes de marché, peuvent stabiliser les conflits de classes et d'autres intérêts sociaux, dans la perspective d'une vie viable.}

errements de la volonté populaire. Ainsi, en Europe, la soumission de l'ordre politique aux dominants de l'ordre économique se profile, sous les masques d'une technocratie qui se pare du masque de la bienveillance ou de la nécessité. Le projet d'une monnaie unique réalisé en dépit de son manque de réalisme, dénoncé par des économistes assez différents ${ }^{22}$, est le symptôme d'une stratégie adoptée par les élites européennes depuis le plan Werner des années 1970: elle consiste en un système économique débarrassé des immixtions de la démocratie dans une ère postfasciste. L'étalonor était l'institution tenant à distance la politique de l'économie à l'époque du capitalisme libéral. Depuis les années 1970, cet idéal de la société de marché s'incarne dans le principe de l'indépendance de la banque centrale, sa mise en place, en Europe, se durcissant avec la mise monnaie unique. 
Polanyi nous avertissait pourtant, dès 1943, que l'étalon-or implique que «les pouvoirs financiers interviennent dans les affaires intérieures de chaque État [...] parce que la coopération traditionnelle est possible uniquement si les systèmes internes sont semblables ${ }^{23}$. Au contraire, l'époque du New Deal «a de nombreux avantages pratiques: il n'est plus nécessaire de contraindre tous les États du monde à rejoindre le lit de Procuste de la fédération, parce que maintenant il est possible pour leurs gouvernements de coopérer librement $»^{24}$. Voilà donc, a contrario, le sens de la monnaie unique européenne : reconstruire le «lit de Procuste» du vieux capitalisme libéral, liquider la souveraineté des peuples au profit de celle du capital, au prix de la vie des nations qui ne correspondent pas aux exigences de ce projet. Mais l'idéologie eurolibérale perdra de sa capacité à organiser le réel si une stagnation longue est le prix à payer pour la perpétuation de la monnaie unique. Plus généralement, la catastrophe nucléaire japonaise de l'année 2011 accroîtra les contraintes pesant sur ce qui a constitué, longtemps, un facteur crucial de légitimation de la société de marché : la croissance. C'en est fini d'une énergie qui était bon marché de façon purement illusoire.

Polanyi, en son temps, avait déjà mis en question la modernité libérale, d'où était issue l'《impasse fasciste ${ }^{25}$. Plus tard, il a opposé la nécessité de l'habitation raisonnée du monde à l'amélioration pourvoyeuse de profit ${ }^{26}$, intitulant un chapitre de la Grande Transformation, «Le marché et la nature», qui se finissait ainsi : «On ne peut séparer nettement les dangers qui menacent l'homme de ceux qui menacent la nature $»^{27}$. La crise de la modernité ne mettait donc pas en cause un seul projet humain (la démocratie sociale contre la société de marché) mais, peut-être, le monde lui-même au-delà de l'homme? La question ici n'était plus de vivre mais de survivre, suite au productivisme impliqué par le Grand Marché.

En 2008, dans le sillage de travaux nombreux, F. Neyrat nous avertissait de ce que la notion de « risque », cœur de l'économie contemporaine, était impuissante à appréhender les déterminations catastrophiques de notre monde, où l'interdépendance croissante entre économie et écosystème vide de sens la notion de «risque naturel ». Au minimum, le risque, qui compte pour l'économie et la société, est absolument non probabilisable : ceci est le cauchemar de la science économique encore dominante. C'est la fin de la logique assurantielle, pivot de l'orthodoxie en économie et de nombres d'institutions économiques, même si une socialisation croissante des coûts privés, indirecte ou directe, masque cette fin de la logique assurantielle

Au minimum, le risque, qui compte pour l'économie et la société, est absolument non probabilisable : ceci est le cauchemar de la science économique encore dominante.

\section{Plusieurs évolutions peuvent être dessinées}

Soit, nous persévérons dans la logique létale de la société de marché ou ses fausses alternatives chinoise ou iranienne ${ }^{28}$.

Soit, face à ces néocapitalismes, nous inventons un néosocialisme fondé sur la primauté de la vie et des liens sociaux, soustrayant définitivement au domaine du marché ce qui n'est pas produit pour être vendu. Il s'agit donc de poser ici la question des limites du marché et de l'appropriation collective des modes de consommation, le vieux socialisme étant fondé sur la centralité de la propriété sociale - en fait étatique - des biens de production ${ }^{29}$.

Faute d'une alternative politique globale, la politique de l'enracinement, la création de communautés, peut se substituer aux interventions de l'État pour ce qui est de la stabilité sociale. Le néolibéralisme trouvera un allié aussi inattendu que solide dans ces «formes d'appartenance à des communautés organiques définies à partir de la parenté, de l'ethnicité et de la religion ${ }^{30}$. L'idéologie du capitalisme mondial est une foire aux identités aux vertus bien conservatrices, ce que ne comprennent pas certains contestataires médiatiques de la société de marché. C'est donc loin de ces capitalismes parés des beaux atours de la différence et fondés sur la réalité de l'indifférence aux autres et à la nature qu'il nous faut penser et agir autrement. 


\section{BIBLIOGRAPHIE ET NOTES}

${ }^{1} \mathrm{Je}$ remercie mon collègue et ami, Claude Gautier, professeur à l'ENS de Lyon, membre également de «Triangle », pour la lecture suggestive qu'il fit d'une première version de ce texte. Je reste seul responsable de la forme finale.

${ }^{2}$ «Il n'existe aucun " déterminisme économique » dans ces conditions » : Karl Polanyi, « Appendice 1. Où va la civilisation? », Institut Karl Polanyi d'économie politique, Montréal, traduction par Laurence Collaud (2004), 1946.

${ }^{3}$ Ibid.

${ }^{4}$ Karl Polanyi, La Grande Transformation, Paris, Gallimard, 1983, p. 259.

${ }^{5}$ Jacques Sapir, Faut-il sortir de l'Euro ?, Paris, le Seuil, 2012.

${ }^{6}$ Polanyi ne le dit pas explicitement.

${ }^{7}$ Karl Polanyi, La Grande Transformation, op. cit., p. 112 et p. 108.

${ }^{8}$ Ibid., p. 257 et p. 262.

${ }^{9}$ Ibid., p. 265.

${ }^{10}$ Karl Polanyi, « Economie et démocratie » (1932), pp. 353-357, dans M. Cangiani, J. Maucourant dir. Essais de Karl Polanyi, Seuil, 2008, p. 354.

${ }^{11}$ En français dans le texte.

${ }^{12}$ Karl Polanyi, La Grande Transformation, op. cit., p. 305 .

${ }^{13}$ Ibid., p. 330.

${ }^{14}$ Karl Polanyi, « L'essence du fascisme » (1935), pp. 369-395, dans M. Cangiani, J. Maucourant dir. Essais de Karl Polanyi, Seuil, 2008, p. 394.

${ }^{15}$ Jérôme Maucourant, Avez-vous lu Polanyi ?, Paris, Flammarion, 2011, p. 205.

${ }^{16}$ Karl Polanyi, « Capitalisme universel ou planification régionale ? », pp. 485-493, dans M. Cangiani, J. Maucourant dir., Essais de Karl Polanyi, Seuil, 2008, p. 491

${ }^{17}$ Karl Polanyi, « Le mécanisme de la crise économique mondiale », pp. 337-351, dans M. Cangiani, J. Maucourant dir., Essais de Karl Polanyi, Seuil, 2008, p. 348.

${ }^{18}$ Karl Polanyi, La Grande Transformation, op. cit., p. 152.

${ }^{19}$ Paul. R. Krugman, (2007) Trade and Inequality, revisited, 15 juin.

${ }^{20}$ Voir le chapitre 4 de Jérôme Maucourant, Avezvous lu Polanyi ?, op. cit.

${ }^{21}$ Voir Jérôme Maucourant et Véronique Taquin, «Lecture : sur R. Reich, Supercapitalisme. Le choc entre le système économique émergent et la démocratie », La Revue du M.A.U.S.S, n ${ }^{\circ}$ 1, 1er sem. 2008, 563-575.

${ }^{23}$ Karl Polanyi cité par Geörgy Litván, « Democratic and socialist thought in Karl Polanyi's thought », pp.
251-271 dans Margie Mendell, Daniel Sallée, The legacy of Karl Polanyi, Londres : Mc Millan, p. 260.

${ }^{24}$ Ibid.

${ }^{25}$ Karl Polanyi, «L'essence du fascisme », pp. 369395, dans M. Cangiani, J. Maucourant dir., op. cit.

${ }^{26}$ « Habitation contre amélioration » est le sous-titre du chapitre 3 de La Grande Transformation.

${ }^{27}$ Karl Polanyi, La Grande Transformation, op. cit., p. 253.

${ }^{28}$ Voir Ramine Motamed-Nejad, « Régimes monétaires, rapports de pouvoir et métamorphoses du capitalisme en Iran (1989-2006) », pp. 209-230, dans E. Lafaye de Michaux, E. Mulot, et Pépita Ould-Ahmed eds., Institutions et développement - la fabrique institutionnelle et politique des trajectoires de développement, Rennes, PUR, 2007.

${ }^{29}$ Jérôme Maucourant, Frédéric Neyrat, « La communauté politique contre le néocapitalisme », pp. 111-139, dans Werner Schönig ed., Perspectiven institutionnalischer Ökonomik, Münster, Lit, 2001

${ }^{30}$ Nous nous inspirons ici de l'analyse de Ayse Bugra, « Karl Polanyi et la séparation institutionnelle entre politique et économie », Raisons politiques études de pensée politique, 20, 2005, pp. 37-55. 